\title{
Effect of Oblateness of the More Massive Primary on Periodic Orbits in the Restricted Three-Body Problem
}

\author{
A. Safiya Beevi ${ }^{1}$ and Ram Krishan Sharma ${ }^{2 *}$ \\ ${ }^{1}$ Department of Mathematics, College of Engineering, Thiruvananthapuram - 695016, India \\ ${ }^{2}$ Department of Aerospace Engineering, Karunya University, Coimbatore - 641114, Tamilnadu, India \\ Email: ramkrishansharma@gmail.com
}

\begin{abstract}
The motion of a particle in the restricted three-body problem is explored by treating the more massive primary as an oblate spheroid with its equatorial plane coincident with the plane of motion of the primaries using a perturbation method. Initial conditions for the infinitesimal periodic orbits around the more massive primary are generated and the effect of oblateness on the perigee of these orbits is studied as well. It is observed that when oblateness coefficient is increased, the perigee of the orbit shifts towards both the primaries depending upon the increase in period and mass ratio. It is further noticed that during this transition, for certain periods, the perigee of the orbit remains unaltered with the increase in the oblateness coefficient.
\end{abstract}

Keywords: RTBP, Earth-Moon System, series solution, periodic orbit, oblateness effect.

\section{Introduction}

Important information about a dynamical system is provided by the study of the periodic orbits in the system. A periodic solution is regarded as a solution of the differential equation of motion that satisfies, in addition to the initial conditions, that after a lapse of one period both coordinates and velocities return to their initial values. The general aspects of periodic orbits in the restricted three- body problem (RTBP) are well known. The works of Stromgren's group at Copenhagen [1] and that of Moulton [2] and Darwin [3] on periodic orbits in RTBP are very important contributions. Broucke [4] had presented a large number of periodic orbits, classified into 10 families, in the Earth-Moon system RTBP. Szebehely [5] provided an excellent treatise on RTBP and discussed various aspects including periodic orbits.

Many of the problems facing physicists, engineers, and applied mathematicians involve difficulties as nonlinear governing equations, variable coefficients, and nonlinear boundary conditions at complex known or unknown boundaries that preclude solving them exactly. Consequently, solutions are approximated using numerical techniques, analytic techniques and combinations of both. New analytical techniques were discovered and became operational, including averaging methods, regularization, Lie series, KAM Theory, canonical operations in the extended phase space, resonance theory, perturbations in rectangular co-ordinates, asymptotic expansions and the theory of singular perturbations, search for new integrals, and new concepts of local and global stability, to mention just a few. Both general and special perturbation techniques benefitted from computer development. Algebraic manipulations in general, computerized Poisson and Fourier series, and compression and storage of ephemerides by Chebyshev polynomials might be mentioned as the most significant non numerical uses of computers assisting the development of general perturbation theories.

Generally in perturbation methods, we start with an integrable system whose solutions are known completely, and study a small perturbation of the unperturbed solution. Since the unperturbed and perturbed vector fields are closed, we might expect that the solutions will also be close. This is not always the case. In that case the unperturbed system is often structurally unstable. Arbitrarily small perturbations of such systems cause radical qualitative changes in the structure of solutions. Foremost, among the analytic techniques are the systematic methods of perturbations (asymptotic expansions) in terms of a small or a large parameter or co-ordinate. McCuskey [6] treated the basic mathematical description of the perturbation problem together with the application to some astronomical systems. The book "Introduction to perturbation methods" by Nayfeh [7] presented in a unified way the most of the perturbation techniques. The book by Guckenheimer and Holms [8] provides the analytical methods 
of averaging and perturbation theory for the study of periodically forced nonlinear oscillators. Various perturbation techniques for differential equations are described by Jordan and Smith [9] in their book "Nonlinear ordinary differential equations". A detailed analysis of the stability properties of periodic orbits, in the cases that are relevant to the operations of electromagnetic tethers working in circular inclined orbits, has been carried out by Pelaez and Lara [10] by using an algorithm based on the Poincare method of continuation of periodic orbits.

In the framework of the planar circular RTBP, Huang [11] used the method of successive approximations to study some interesting orbits which provided a useful background for deriving periodic orbits for the Moon probing vehicle in the Earth-Moon-Sun system. Huang and Wade [12] derived two families of direct and retrograde periodic orbits that enclose both the Earth and the Moon. Their stability was examined by investigating the variations of the difference of two successive periods. Later Huang [13] established a series solution for periodic orbits in the RTBP which were revolving around the more massive primary. Recently Abouelmagd et al. [14] studied periodic and secular solutions in the RTBP under the effect of zonal harmonics of the more massive primary around the triangular points.

In this paper, we study the periodic orbits in the RTBP by Huang's method by considering the more massive primary as an oblate spheroid with its equatorial plane coincident with the plane of motion. Two parameters, the mass ratio $\mu=m_{2} /\left(m_{1}+m_{2}\right)$, where $m_{1}$ and $m_{2}$ are masses of the more massive and smaller primary, respectively, and the oblateness coefficient $A_{1}=\left(A E^{2}-A P^{2}\right) / 5 R^{2}$, where $A E$ and $A P$ are the equatorial and polar radii of the more massive primary, and $R$ is the distance between the primaries, are chosen for series expansions. Initial conditions for the infinitesimal periodic orbits around the more massive primary are generated and the effect of oblateness on the perigee of the generated orbits is studied as well.

The paper is organized in 8 sections. Section 2 deals with the equations of motion with origin at the more massive primary. The equations of motion in polar co-ordinates are described in Section 3. Equations of perturbations are provided in Section 4. In Section 5, second order equations of motion with approximations are given. A solution of first order equations is presented in Section 6. Numerical applications are shown in Section 7 and the conclusions are drawn in Section 8.

\section{$2 \quad$ Equations of Motion}

The equations of motion of the problem under consideration in the dimensionless barycentric synodic coordinate system $\left(x_{1}, x_{2}\right)$ are (Sharma and Subba Rao [15])

$$
\ddot{x}_{1}-2 n \dot{x}_{2}=\frac{\partial \Omega}{\partial x_{1}}, \quad \ddot{x}_{2}+2 n \dot{x}_{1}=\frac{\partial \Omega}{\partial x_{2}},
$$

with

$$
r_{1}=\left(\left(x_{1}-\mu\right)^{2}+x_{2}^{2}\right)^{1 / 2}, \quad r_{2}=\left(\left(x_{1}+1-\mu\right)^{2}+x_{2}^{2}\right)^{1 / 2}
$$

and

$$
n^{2}=1+\frac{3}{2} A_{1}
$$

where

$$
\Omega=\frac{n^{2}}{2}\left(x_{1}^{2}+x_{2}^{2}\right)+\frac{1-\mu}{r_{1}}+\frac{\mu}{r_{2}}+\frac{A_{1}(1-\mu)}{2 r_{1}^{3}} .
$$

Shifting the origin to $m_{1}$, the equations of motion become

$$
\frac{\mathrm{d}^{2} x_{1}}{\mathrm{~d} t^{2}}-2 n \frac{\mathrm{d} x_{2}}{\mathrm{~d} t}-n^{2}\left(x_{1}+\mu\right)=-\left(\frac{(1-\mu) x_{1}}{r_{1}^{3}}+\frac{\mu\left(x_{1}+1\right)}{r_{2}^{3}}+\frac{3 A_{1}(1-\mu) x_{1}}{2 r_{1}^{5}}\right)
$$




$$
\frac{\mathrm{d}^{2} x_{2}}{\mathrm{~d} t^{2}}+2 n \frac{\mathrm{d} x_{1}}{\mathrm{~d} t}-n^{2} x_{2}=-\left(\frac{(1-\mu) x_{2}}{r_{1}^{3}}+\frac{\mu x_{2}}{r_{2}^{3}}+\frac{3 A_{1}(1-\mu) x_{2}}{2 r_{1}^{5}}\right)
$$

where

$$
r_{1}=\left(x_{1}^{2}+x_{2}^{2}\right)^{1 / 2}, \quad r_{2}=\left(\left(x_{1}+1\right)^{2}+x_{2}^{2}\right)^{1 / 2},
$$

and

$$
\Omega=\frac{n^{2}}{2}\left(x_{1}^{2}+x_{2}^{2}\right)+\frac{1-\mu}{r_{1}}+\frac{\mu}{r_{2}}+\frac{A_{1}(1-\mu)}{2 r_{1}^{3}} .
$$

\section{Equation of Motion in Polar Coordinates}

In the equations of motion, it is assumed that the mass $1-\mu$ is at the origin and the mass $\mu$ is at the point $(-1,0)$. Also $r_{1}, r_{2}$ are the distances of the third body from the masses $1-\mu$ and $\mu$, respectively. So $r_{1}$ is the distance of the third body from the origin. Let $r_{1}=r$. We use the transformation

$$
\begin{aligned}
& x_{1}=r \cos \theta \\
& x_{2}=r \sin \theta
\end{aligned}
$$

This converts the Equations (2.1) and (2.2) into the form

$$
\begin{gathered}
\frac{\mathrm{d}^{2} r}{\mathrm{~d} t^{2}}-r\left(\frac{\mathrm{d} \theta}{\mathrm{d} t}\right)^{2}-2 n r \frac{\mathrm{d} \theta}{\mathrm{d} t}=n^{2} r-\frac{(1-\mu)}{r^{2}}-\frac{\mu r}{r^{3}}-\frac{3 A_{1}(1-\mu)}{2 r^{4}}+\mu\left(n^{2}-\frac{1}{r_{2}^{3}}\right) \cos \theta \\
r \frac{\mathrm{d}^{2} \theta}{\mathrm{d} t^{2}}+2 \frac{\mathrm{d} r}{\mathrm{~d} t} \frac{\mathrm{d} \theta}{\mathrm{d} t}+2 n \frac{\mathrm{d} r}{\mathrm{~d} t}=-\mu\left(n^{2}-\frac{1}{r_{2}^{3}}\right) \sin \theta
\end{gathered}
$$

\section{Equations of Perturbations}

When $\mu$ is equal to zero, all circles with centre at the origin are the periodic solutions of the problem. If $\mu$ is small, the periodic orbits deviate only slightly from the circular one. The deviation depends upon $\mu$. Therefore, the periodic solution may be written as

$$
\begin{aligned}
& r=r_{0}+\mu r_{11}(t)+\mu^{2} r_{22}(t)+\ldots \ldots \ldots \\
& \theta=\lambda t+\mu \theta_{1}(t)+\mu^{2} \theta_{2}(t)+\ldots \ldots \ldots
\end{aligned}
$$

where $r_{0}$ and $\lambda$ are to be determined and are independent of time. Substitution of Equations (4.1) and (4.2) into Equations (3.3) and (3.4) provides different order of approximations.

The zeroth $\left(\mu^{0}\right)$ order approximation gives

$$
(\lambda+n)^{2}=\frac{1-\mu}{r_{0}^{3}}
$$

which is Kepler's third law in the problem of two bodies. The term is $n+\lambda$ instead of $\lambda$ because the equations are expressed in rotating co-ordinate system. Also $\lambda$ may have positive or negative value corresponding to the third body. Neglecting higher order terms in $A_{1}$, we get

$$
(\lambda+1)^{2}+\frac{3}{2} A_{1}(\lambda+1)=\frac{1-\mu}{r_{0}^{3}}
$$

If the terms involving third and higher orders of $r_{0}$ are neglected, the first order approximation yields 


$$
\begin{gathered}
\frac{\mathrm{d}^{2} r_{11}}{\mathrm{~d} t^{2}}-2(\lambda+n) r_{0} \frac{\mathrm{d} \theta_{1}}{\mathrm{~d} t}=\left(\begin{array}{c}
3(\lambda+n)^{2} r_{11} \\
+6 \frac{r_{11} A_{1}}{r_{0}^{5}}+\frac{r_{0}}{2}+\frac{3 A_{1}}{2 r_{0}^{4}}+\left(n^{2}-1-\frac{9}{8} r_{0}^{2}\right) \cos \lambda \\
+\frac{3}{2} r_{0} \cos 2 \lambda t-\frac{15}{8} r_{0}^{2} \cos 3 \lambda t
\end{array}\right) \\
r_{0} \frac{\mathrm{d}^{2} \theta_{1}}{\mathrm{~d} t^{2}}+2(\lambda+n) \frac{\mathrm{d} r_{11}}{\mathrm{~d} t}=\left(1-n^{2}+\frac{3}{8} r_{0}^{2}\right) \sin \lambda t-\frac{3}{2} r_{0} \sin 2 \lambda t-\frac{15}{8} r_{0}^{2} \sin 3 \lambda t
\end{gathered}
$$

Neglecting higher order terms in $A_{1}$ provides

$$
\begin{gathered}
\frac{\mathrm{d}^{2} r_{11}}{\mathrm{~d} t^{2}}-2\left(\lambda+1+\frac{3}{4} A_{1}\right) r_{0} \frac{\mathrm{d} \theta_{1}}{\mathrm{~d} t}=\left(\begin{array}{c}
3\left((\lambda+1)^{2}+\frac{3}{2} A_{1}(\lambda+1)\right) r_{11} \\
+6 \frac{r_{11} A_{1}}{r_{0}^{5}}+\frac{r_{0}}{2}+\frac{3 A_{1}}{2 r_{0}^{4}}+\left(\frac{3}{2} A_{1}-\frac{9}{8} r_{0}^{2}\right) \cos \lambda t \\
+\frac{3}{2} r_{0} \cos 2 \lambda t-\frac{15}{8} r_{0}^{2} \cos 3 \lambda t
\end{array}\right) \\
r_{0} \frac{\mathrm{d}^{2} \theta_{1}}{\mathrm{~d} t^{2}}+2\left(\lambda+1+\frac{3}{4} A_{1}\right) \frac{\mathrm{d} r_{11}}{\mathrm{~d} t}=\left(\begin{array}{c}
\left(-\frac{3}{2} A_{1}+\frac{3}{8} r_{0}^{2}\right) \sin \lambda t-\frac{3}{2} r_{0} \sin 2 \lambda t \\
-\frac{15}{8} r_{0}^{2} \sin 3 \lambda t
\end{array}\right)
\end{gathered}
$$

\section{$5 \quad$ Second Order Equations}

With the same degree of approximation in regard to the series in $r_{0}$, the second order $\left(\mu^{2}\right)$ equations are

$$
\begin{aligned}
& \frac{\mathrm{d}^{2} r_{22}}{\mathrm{~d} t^{2}}-2(\lambda+n) r_{0} \frac{\mathrm{d} \theta_{2}}{\mathrm{~d} t}-3 r_{22}(\lambda+n)^{2}-r_{0}\left(\frac{\mathrm{d} \theta_{1}}{\mathrm{~d} t}\right)^{2}-2(\lambda+n) r_{11} \frac{\mathrm{d} \theta_{1}}{\mathrm{~d} t} \\
& =\left(\begin{array}{c}
-3(\lambda+n)^{2} \frac{r_{11}^{2}}{r_{0}}+\frac{r_{11}}{2}-\frac{9}{4} r_{0} r_{11} \cos \lambda t+\frac{3}{2} r_{11} \cos 2 \lambda t-\frac{15}{4} r_{0} r_{11} \cos 3 \lambda t \\
+6 r_{0} r_{11} A_{1}(\lambda+n)^{2}+\frac{3 A_{1}}{2 r_{0}}(\lambda+n)^{2}+6 r_{0} r_{22} A_{1}(\lambda+n)^{4}-6 r_{0} r_{11} A_{1}(\lambda+n)^{4} \\
-\left(n^{2}-1+\frac{9}{8} r_{0}^{2}\right) \theta_{1} \sin \lambda t-3 r_{0} \theta_{1} \sin 2 \lambda t+\frac{45}{8} r_{0}^{2} \theta_{1} \sin 3 \lambda t
\end{array}\right) \\
& r_{0} \frac{d^{2} \theta_{2}}{d t^{2}}+2(\lambda+n) \frac{d r_{22}}{d t}+2 \frac{d r_{11}}{d t} \frac{d \theta_{1}}{d t}+r_{11} \frac{d^{2} \theta_{1}}{d t^{2}}=\left(\begin{array}{c}
\frac{3}{4} r_{0} r_{11} \sin \lambda t+\frac{3}{2} r_{11} \sin 2 \lambda t \\
+\frac{15}{4} r_{0} r_{11} \sin 3 \lambda t+\left(1-n^{2}+\frac{3}{8} r_{0}^{2}\right) \theta_{1} \cos \lambda t \\
-3 r_{0} \theta_{1} \cos 2 \lambda t+\frac{45}{8} r_{0}^{2} \theta_{1} \cos 3 \lambda t
\end{array}\right)
\end{aligned}
$$

Similarly the equations of higher orders can be derived in terms of the solutions of the equations of the lower orders.

\section{Solutions of First Order Equations}

Differentiating equation (4.7) with respect to $t$ and substituting equation (4.8) in the resulting expression, we get 


$$
\frac{\mathrm{d}^{3} r_{11}}{\mathrm{~d} t^{3}}+p^{2} \frac{\mathrm{d} r_{11}}{\mathrm{~d} t}=p_{1} \sin \lambda t+p_{2} \sin 2 \lambda t+p_{3} \sin 3 \lambda t
$$

where

Solving Equation (6.1), we get

$$
\begin{gathered}
p^{2}=(\lambda+n)^{2}\left(1-\frac{6 A_{1}}{r_{0}^{2}(1-\mu)}\right) \\
p_{1}=\frac{3}{8} r_{0}^{2}(5 \lambda+2 n)+(3 \lambda+2 n)\left(1-n^{2}\right) \\
p_{2}=-3 r_{0}(2 \lambda+n) \\
p_{3}=\frac{15}{8} r_{0}^{2}(5 \lambda+2 n)
\end{gathered}
$$

$$
r_{11}=c_{0}+c_{1} \cos p t+c_{2} \sin p t+k_{1} \cos \lambda t+k_{2} \cos 2 \lambda t+k_{3} \cos 3 \lambda t
$$

where $c_{0}, c_{1}, c_{2}$ are arbitrary constants and

$$
\begin{gathered}
k_{1}=-\frac{p_{1}}{\lambda\left(p^{2}-\lambda^{2}\right)} \\
k_{2}=-\frac{p_{2}}{2 \lambda\left(p^{2}-4 \lambda^{2}\right)} \\
k_{3}=-\frac{p_{3}}{3 \lambda\left(p^{2}-9 \lambda^{2}\right)}
\end{gathered}
$$

Substituting (6.6) in Equation (4.8), we get

Integrating, we get

$$
r_{0} \frac{\mathrm{d}^{2} \theta_{1}}{\mathrm{~d} t^{2}}=\left(\begin{array}{c}
2(\lambda+n) p c_{1} \sin p t-2(\lambda+n) p c_{2} \cos p t \\
+\left(2(\lambda+n) \lambda k_{1}+1-n^{2}+\frac{3}{8} r_{0}^{2}\right) \sin \lambda t \\
+\left(4(\lambda+n) \lambda k_{2}-3 \frac{r_{0}}{2}\right) \sin 2 \lambda t \\
+\left(6(\lambda+n) \lambda k_{3}+\frac{15}{8} r_{0}^{2}\right) \sin 3 \lambda t
\end{array}\right)
$$

where

$$
r_{0} \theta_{1}=D_{1} \sin p t+D_{2} \cos p t+l_{1} \sin \lambda t+l_{2} \sin 2 \lambda t+l_{3} \sin 3 \lambda t+B_{1} t+B_{2}
$$

where $D_{1}, D_{2}$ depend on $c_{1}, c_{2} . B_{1}, B_{2}$ are arbitrary constants. 
Substituting the values of $r_{11}, \theta_{1}$ in (4.7) and equating constant terms, we get

$$
c_{0}=\left(\begin{array}{c}
-\frac{A_{1}}{2 r_{0}}-\frac{r_{0}}{6(\lambda+1)^{2}}\left(1-\left(\frac{2}{r_{0}^{2}(1-\mu)}+\frac{3}{2(\lambda+1)}\right) A_{1}-\frac{2 B_{1}}{3(\lambda+1)}\right) \\
\times\left(1-\left(\frac{2}{r_{0}^{2}(1-\mu)}+\frac{3}{4(\lambda+1)}\right) A_{1}\right)
\end{array}\right)
$$

Thus neglecting the higher powers of $A_{1}$, the solution of (4.7) and (4.8) is obtained as

$$
r_{11}=\left(\begin{array}{l}
-\frac{A_{1}}{2 r_{0}}-\frac{r_{0}}{6(\lambda+1)^{2}}\left(1-\left(\frac{2}{r_{0}^{2}(1-\mu)}+\frac{3}{2(\lambda+1)}\right) A_{1}-\frac{2 B_{1}}{3(\lambda+1)}\right) \\
\times\left(1-\left(\frac{2}{r_{0}^{2}(1-\mu)}+\frac{3}{4(\lambda+1)}\right) A_{1}\right)+c_{1} \cos p t+c_{2} \sin p t+k_{1} \cos \lambda t+k_{2} \cos 2 \lambda t+k_{3} \cos 3 \lambda t
\end{array}\right)
$$

and

$$
r_{0} \theta_{1}=D_{1} \sin p t+D_{2} \cos p t+l_{1} \sin \lambda t+l_{2} \sin 2 \lambda t+l_{3} \sin 3 \lambda t+B_{1} t+B_{2}
$$

The values of $p, k_{1}, k_{2}, k_{3}, l_{1}, l_{2}, l_{3}$ are given by

$$
\begin{aligned}
& p^{2}=(\lambda+1)^{2}+\frac{3}{2} A_{1}(\lambda+1)\left(1-\frac{4(\lambda+1)}{r_{0}^{2}(1-\mu)}\right) \\
& k_{1}=-\frac{3(5 \lambda+2)}{8 \lambda(2 \lambda+1)} r_{0}^{2}+\frac{A_{1}}{\lambda}\left(\frac{9}{16} \frac{5 \lambda^{2}+5 \lambda+1}{(2 \lambda+1)^{2}} r_{0}^{2}+\frac{3(3 \lambda+2)}{(2 \lambda+1)}-\frac{9}{4} \frac{(5 \lambda+2)(\lambda+1)^{2}}{(2 \lambda+1)^{2}(1-\mu)}\right) \\
& k_{2}=-\frac{3(2 \lambda+1)}{2 \lambda\left(3 \lambda^{2}-2 \lambda-1\right)} r_{0}-\frac{9 A_{1}}{\lambda\left(3 \lambda^{2}-2 \lambda-1\right)^{2}}\left(\frac{\left(7 \lambda^{2}+4 \lambda+1\right)}{8} r_{0}-\frac{(2 \lambda+1)(\lambda+1)^{2}}{r_{0}(1-\mu)}\right) \\
& k_{3}=\frac{5(5 \lambda+2)}{8 \lambda\left(8 \lambda^{2}-2 \lambda-1\right)} r_{0}^{2}+\frac{15 A_{1}}{\lambda\left(8 \lambda^{2}-2 \lambda-1\right)^{2}}\left(\frac{\left(3 \lambda^{2}-9 \lambda+3\right)}{16} r_{0}^{2}+\frac{(5 \lambda+2)(\lambda+1)^{2}}{4(1-\mu)}\right) \\
& l_{1}=\frac{3\left(10 \lambda^{2}+12 \lambda+3\right)}{8 \lambda^{2}(2 \lambda+1)} r_{0}^{2}+\frac{9 A_{1}}{\lambda^{2}(2 \lambda+1)^{2}}\left(\begin{array}{c}
\frac{1}{16}\left(\begin{array}{c}
-10 \lambda^{3}+122 \lambda^{2} \\
+147 \lambda+42
\end{array}\right) r_{0}^{2} \\
+\frac{\left(5 \lambda^{2}-3 \lambda-3\right)(\lambda+1)^{2}}{2(1-\mu)} \\
-\frac{3}{2}\left(6 \lambda^{2}+8 \lambda+3\right)(2 \lambda+1)
\end{array}\right) \\
& l_{2}=\frac{3\left(11 \lambda^{2}+10 \lambda+3\right)}{8 \lambda^{2}\left(3 \lambda^{2}-2 \lambda-1\right)} r_{0}+\frac{9 A_{1}(\lambda+1)}{8 \lambda^{2}\left(3 \lambda^{2}-2 \lambda-1\right)^{2}}\left(\begin{array}{c}
\left(7 \lambda^{2}+4 \lambda+1\right) r_{0} \\
-\frac{2\left(11 \lambda^{2}+10 \lambda+3\right)(\lambda+1)}{r_{0}(1-\mu)}
\end{array}\right) \\
& l_{3}=-\frac{5\left(6 \lambda^{2}+4 \lambda+1\right)}{8 \lambda^{2}\left(8 \lambda^{2}-2 \lambda-1\right)} r_{0}^{2}-\frac{5 A_{1}(\lambda+1)}{\lambda^{2}\left(8 \lambda^{2}-2 \lambda-1\right)^{2}}\left(\begin{array}{c}
\frac{3}{16}\left(\begin{array}{l}
38 \lambda^{3}+26 \lambda^{2} \\
-5 \lambda-2
\end{array}\right) r_{0}^{2} \\
-\frac{\left(29 \lambda^{2}+\lambda-1\right)(\lambda+1)^{2}}{2(1-\mu)}
\end{array}\right)
\end{aligned}
$$


The periodic orbits around the more massive primary is given up to the first order terms in $r_{0}$ and $\mu$ by

and

$$
r=r_{0}+\mu r_{11}
$$

$$
\theta=\lambda t+\mu \theta_{1}
$$

where

$$
\left.r_{11}=-\frac{r_{0}}{6(\lambda+1)^{2}}\left(\begin{array}{c}
\left.\left(1-\left(\frac{2}{r_{0}^{2}(1-\mu)}+\frac{3}{2(\lambda+1)}\right) A_{1}-\frac{2 B_{1}}{3(\lambda+1)}\right) \times\right) \\
+k_{1} \cos \lambda t+k_{2} \cos 2 \lambda t+k 3_{1} \cos 3 \lambda t \\
r_{0}^{2}(1-\mu)
\end{array}\right)-\frac{3}{4(\lambda+1)}\right)
$$

and

$$
r_{0} \theta_{1}=l_{1} \sin \lambda t+l_{2} \sin 2 \lambda t+l_{3} \sin 3 \lambda t
$$

It follows from the solution given by Equations (6.18) and (6.19) that in general, a periodic solution can be obtained for any given value of $\lambda$ only by setting $c_{1}, c_{2}, B_{1}, B_{2}$ equal to zero. Thus one periodic orbit is associated with one value of the period. All the foregoing relations with $A_{1}=0$ are the same as in Huang (1964), except for sign changes at some places due to the difference in the utilized co-ordinate system.

\section{Numerical Applications}

The Earth-Moon system is considered to obtain periodic orbits using Equations (5.28) and (5.29). In the $x_{1}-x_{2}$ co-ordinate system with origin at the mass $(1-\mu)$, having $\mu=0.012149, A_{1}=0$ and period $T$ $=0.23802754$, the value of $\lambda$ is obtained from the expression $T=2 \pi / \lambda$ as 26.396884. Substituting these values in Equation (4.4) we get $r_{0}$. The values of $k_{n}, l_{n}$ are calculated using Equations (5.21) to (5.26), which are shown in Table 1. Substituting these values in Equations (5.27) to (5.30) and transforming the variables from $r$ and $\theta$ to $x_{1}$ and $x_{2}$ in accordance with Equations (3.1) and (3.2) will give the values of $x_{1}, x_{2}, \dot{x}_{1}, \dot{x}_{2}$ when $t=0$, which are denoted by $x_{0}, y_{0}, \dot{x}_{0}, \dot{y}_{0}$. For various values of period $T$, we have calculated $x_{0}, y_{0}, \dot{x}_{0}, \dot{y}_{0}$ and $C$, which are presented in Table 2 . The values are compared with Huang's results.

Similarly, the Sun-Jupiter system is considered to derive periodic orbits, with $\mu=0.0009539, A_{1}=0$, and period $T=0.23802754$. The values of $k_{n}, l_{n}$ are calculated, which are shown in Table 3 . For various values of period, $x_{0}, y_{0}, \dot{x}_{0}, \dot{y}_{0}$ and $C$ are calculated and are presented in Table 4 . Using the systems considered in Sharma and Subba Rao $([15], 1976)$, the values of $x_{0}, \dot{y}_{0}$ for periodic orbits around the (respective) more massive primaries are derived, and are shown in Table 5.

Table 1. Value of $k_{n}, l_{n}$ for $T=0.23802754, \lambda=26.396884$.

\begin{tabular}{llll}
\hline$k_{\mathrm{n}}$ & 0.0004249394 & 0.0001644862 & 0.000006901187 \\
\hline$l_{\mathrm{n}}$ & 0.0008756117 & 0.0002296953 & 0.000008365787 \\
\hline
\end{tabular}


Table 2. Value of $x_{0}, y_{0}, \dot{x}_{0}, \dot{y}_{0}(\mu=0.012149)$.

\begin{tabular}{crrccc}
\hline$T$ & $x_{0}$ & $y_{0}$ & $\dot{x}_{0}$ & $\dot{y}_{0}$ & $C$ \\
\hline 0.23802754 & 0.10959079 & 0.000000 & 0.000000 & 2.89272981 & 9.69689124 \\
0.39999890 & 0.15239387 & 0.000000 & 0.000000 & 2.39363721 & 7.28328121 \\
0.59999669 & 0.19580806 & 0.000000 & 0.000000 & 2.05037461 & 5.94990225 \\
0.79999292 & 0.23271671 & 0.000000 & 0.000000 & 1.82777788 & 5.22927567 \\
0.99999028 & 0.26506832 & 0.000000 & 0.000000 & 1.66576735 & 4.77580660 \\
1.19999397 & 0.29394939 & 0.000000 & 0.000000 & 1.53981183 & 4.46403690 \\
1.40001535 & 0.32005209 & 0.000000 & 0.000000 & 1.43763350 & 4.23681317 \\
1.60007155 & 0.34385492 & 0.000000 & 0.000000 & 1.35226205 & 4.06419053 \\
\hline
\end{tabular}

Table 3. Value of $k_{n}, l_{n}$ for $T=0.23802754, \lambda=26.396884, \mu=0.0009539$

\begin{tabular}{lrrr}
\hline$k_{n}$ & -0.0004281438 & -0.0001651052 & 0.000006953228 \\
$l_{n}$ & 0.0008822146 & 0.0002305597 & -0.000008428873 \\
\hline
\end{tabular}

Table 4. Value of $x_{0}, y_{0}, \dot{x}_{0}, \dot{y}_{0}(\mu=0.0009539)$.

\begin{tabular}{cccccc}
\hline$T$ & $x_{0}$ & $y_{0}$ & $\dot{x}_{0}$ & $\dot{y}_{0}$ & $C$ \\
\hline 0.23802754 & -0.11000065 & 0.0000 & 0.0000 & -2.90366433 & 9.74713571 \\
0.39999890 & -0.15296086 & 0.0000 & 0.0000 & -2.40269691 & 7.31517351 \\
0.59999666 & -0.19653370 & 0.0000 & 0.0000 & -2.05809668 & 5.97152838 \\
0.79999290 & -0.23358009 & 0.0000 & 0.0000 & -1.83455031 & 5.24523535 \\
0.99999026 & -0.26605988 & 0.0000 & 0.0000 & -1.67174119 & 4.78809624 \\
1.1999940 & -0.29506812 & 0.0000 & 0.0000 & -1.54503453 & 4.47370815 \\
1.4000154 & -0.32130451 & 0.0000 & 0.0000 & -1.44209402 & 4.24448737 \\
1.6000716 & -0.34525482 & 0.0000 & 0.0000 & -1.35590940 & 4.07026362 \\
\hline
\end{tabular}

Table 5. Value of $x_{0}, \dot{y}_{0}$ (various mass ratios with $T=0.23802754$ ).

\begin{tabular}{cccc}
\hline & $\mu$ & $x_{0}$ & $\dot{y}_{0}$ \\
\hline 1 & 0.0121490000 & -0.10959079 & -2.89272981 \\
2 & 0.0002461294 & -0.11002645 & -2.90435293 \\
3 & 0.0000807835 & -0.11003248 & -2.90451375 \\
4 & 0.0000479677 & -0.11003368 & -2.90454567 \\
5 & 0.0000415283 & -0.11003391 & -2.90455193 \\
6 & 0.0000250794 & -0.11003451 & -2.90456793 \\
7 & 0.0000039400 & -0.11003528 & -2.90458849 \\
8 & 0.0000032000 & -0.11003531 & -2.90458921 \\
9 & 0.0000020390 & -0.11003535 & -2.90459034 \\
10 & 0.0000010950 & -0.11003539 & -2.90459126 \\
11 & 0.0000002000 & -0.11003542 & -2.90459213 \\
12 & 0.0000001480 & -0.11003542 & -2.90459218 \\
13 & 0.0000000659 & -0.11003542 & -2.90459226 \\
14 & 0.0000000520 & -0.11003542 & -2.90459227 \\
\hline
\end{tabular}

In order to study the effect of mass ratio on the periodic orbits, initial conditions are derived for periodic orbits around the more massive primary with mass ratios varying from 0.01 to 0.4 . It is observed that for a given period, the value of $x_{0}$ decreases as mass ratio increases (Table 6). This shows that the perigee of the orbit moves towards the more massive primary as the mass ratio increases. 
Considering different mass ratios, if the oblateness coefficient is increased, the value of $x_{0}$ decreases for a small period. When period is also increased, value of $x_{0}$ decreases for small mass ratios with the increase in the oblateness effect. For mass ratios from 0.015 onwards, the value of $x_{0}$ increases due to the increase in the oblateness. Values of the oblateness coefficient considered are $A_{1}=0.0, A_{11}=$ $0.000001, A_{12}=0.000005, A_{13}=0.00001, A_{14}=0.00005, A_{15}=0.0001, A_{16}=0.0005, A_{17}=0.001, A_{18}=$ 0.005, $A_{19}=0.01$. As an example, we have considered two cases with $T=0.23802754$ and $T=$ 0.59999666. In the first case as oblateness increases, the value of $x_{0}$ decreases for all the mass ratios considered in Table 6. But in the second case, when oblateness increases, the value of $x_{0}$ increases for mass ratios considered from 0.015 onwards (Table 7 ). With further increase in period and oblateness, the value of $x_{0}$ decreases. Perigee variations (in kilometers) are shown in Table 8 and Table 9. Perigee of the orbit moves towards or away from the more massive primary depending on the mass ratio, period and oblateness effect, which is shown in Figures 1 and 2.

Table 6. The value of $x_{0}$ for various oblateness coefficients $(T=0.23802754)$.

\begin{tabular}{cccccccc}
\hline$\mu$ & $A_{1}$ & $A_{11}$ & $A_{12}$ & $A_{13}$ & $A_{14}$ & $A_{15}$ & $A_{16}$ \\
\hline 0.01 & -0.109669709 & -0.109669691 & -0.109669618 & -0.109669527 & -0.109668799 & -0.10966789 & -0.109660613 \\
0.012149 & -0.109590787 & -0.109590766 & -0.109590679 & -0.109590571 & -0.109589706 & -0.109588624 & -0.109579973 \\
0.015 & -0.109485904 & -0.109485878 & -0.109485773 & -0.109485642 & -0.109484596 & -0.109483287 & -0.109472820 \\
0.02 & -0.109301461 & -0.109301427 & -0.109301291 & -0.109301121 & -0.109299758 & -0.109298055 & -0.109284429 \\
0.03 & -0.108930641 & -0.108930591 & -0.108930393 & -0.108930145 & -0.108928160 & -0.108925679 & -0.108905834 \\
0.05 & -0.108181104 & -0.10818102 & -0.108180706 & -0.108180307 & -0.108177120 & -0.108173136 & -0.108141262 \\
0.1 & -0.106259018 & -0.106258870 & -0.106258277 & -0.106257536 & -0.106251610 & -0.106244202 & -0.106184933 \\
0.2 & -0.102185281 & -0.102185033 & -0.102184039 & -0.102182797 & -0.102172861 & -0.102160442 & -0.102061081 \\
0.3 & -0.097750073 & -0.097749796 & -0.097748688 & -0.097747302 & -0.097736221 & -0.097722368 & -0.097611543 \\
0.4 & -0.092863436 & -0.092863246 & -0.092862488 & -0.092861541 & -0.092853959 & -0.092844482 & -0.092768657 \\
\hline
\end{tabular}

Table 7. The value of $x_{0}$ for various oblateness coefficients $(T=0.59999666)$.

\begin{tabular}{lccccccc}
\hline \multicolumn{1}{c}{$\mu$} & $A_{1}$ & $A_{11}$ & $A_{12}$ & $A_{13}$ & $A_{14}$ & $A_{15}$ & $A_{16}$ \\
\hline 0.01 & -0.195947808 & -0.195947805 & -0.195947795 & -0.195947782 & -0.195947679 & -0.195947551 & -0.195946521 \\
0.012149 & -0.195808055 & -0.195808054 & -0.195808049 & -0.195808043 & -0.195807994 & -0.195807934 & -0.195807447 \\
0.015 & -0.195622312 & -0.195622313 & -0.195622315 & -0.195622318 & -0.195622343 & -0.195622374 & -0.195622622 \\
0.02 & -0.195295624 & -0.195295628 & -0.195295644 & -0.195295664 & -0.195295822 & -0.195296019 & -0.195297598 \\
0.03 & -0.194638634 & -0.194638645 & -0.194638689 & -0.194638744 & -0.194639185 & -0.194639735 & -0.194644140 \\
0.04 & -0.193309916 & -0.193309943 & -0.193310050 & -0.193310185 & -0.193311259 & -0.193312601 & -0.193323341 \\
0.1 & -0.189898224 & -0.189898301 & -0.189898611 & -0.189898998 & -0.189902094 & -0.189905964 & -0.189936924 \\
0.2 & -0.182648652 & -0.182648888 & -0.182649835 & -0.182651018 & -0.1826604850 & -0.182672318 & -0.182766979 \\
0.3 & -0.174731535 & -0.174732040 & -0.174734057 & -0.174736579 & -0.1747567530 & -0.17478197 & -0.174983703 \\
\hline
\end{tabular}

In the case of fixed mass ratio (higher values), if the period is increased to a particular stage, the value of $x_{0}$ increases with oblateness. As the period is increased further, the value of $x_{0}$ decreases due to the increase in oblateness (Table 10) and this phenomenon continues. From Figures 3 and 4 , it is observed that as oblateness coefficient increases, the perigee of the orbit shifts towards both the primaries. It is also observed that during this transition, for certain periods, the perigee of the orbit remains unaltered with the increase in oblateness coefficient (Table 11). Table 12 shows that difference in $y_{0}$ increases as oblateness increases and for a fixed oblateness coefficient; this difference decreases as period increases to a particular value. On further increase in period, this difference increases. For this study, the period considered are $T_{1}=0.23802754, T_{2}=0.039999890, T_{3}=0.59999666, T_{4}=0.79999290$, $T_{5}=0.99999026, T_{6}=1.1999940, T_{7}=1.4000154$ and $T_{8}=1.6000716$. For convenience, symbols $T_{19}: T$ $=0.68419, T_{20}: T=0.68420, T_{201}: T=0.684201$ etc. are used here. 
Table 8. Perigee variations for $T=0.23802754$ (each deviation multiplied by 3840000 )

\begin{tabular}{lccccccccc}
\hline \multicolumn{1}{c}{$\mu$} & $A_{11}$ & $A_{12}$ & $A_{13}$ & $A_{14}$ & $A_{15}$ & $A_{16}$ & $A_{17}$ & $A_{18}$ & $A_{19}$ \\
\hline 0.01 & 0 & 0 & 0 & 0 & 0 & 0 & 0 & 0 & 0 \\
0.012149 & 0.013056 & 0.065664 & 0.131712 & 0.659712 & 1.319808 & 6.599808 & 13.200768 & 66.018048 & 132.07104 \\
0.015 & 0.030336 & 0.152832 & 0.305664 & 1.531008 & 3.062400 & 15.313152 & 30.627840 & 153.172992 & 306.425856 \\
0.02 & 0.061056 & 0.304512 & 0.609024 & 3.047424 & 6.094848 & 30.475008 & 60.951936 & 304.823424 & 609.806208 \\
0.03 & 0.120576 & 0.603200 & 1.206144 & 6.032640 & 12.065200 & 60.328320 & 120.660096 & 603.429120 & 1207.178496 \\
0.05 & 0.23616 & 1.180416 & 2.360832 & 11.80608 & 23.61216 & 118.065024 & 236.136576 & 1180.941312 & 2362.527360 \\
0.1 & 0.49920 & 2.495616 & 4.990848 & 24.955008 & 49.910016 & 249.556992 & 499.128960 & 2496.235008 & 4993.947264 \\
0.2 & 0.883968 & 4.419840 & 8.839680 & 44.198784 & 88.397568 & 442.001664 & 884.036352 & 4421.476224 & 8846.192640 \\
0.3 & 0.994176 & 4.970112 & 9.93984 & 49.700352 & 99.401088 & 497.027328 & 994.106880 & 4972.598784 & 9950.362752 \\
0.4 & 0.657792 & 3.289344 & 6.579072 & 32.898816 & 65.79840 & 329.022720 & 658.119552 & 3293.516928 & 6594.33408 \\
\hline
\end{tabular}

Table 9. Perigee variations for $T=0.59999666$ (each deviation multiplied by 3840000 ).

\begin{tabular}{cccccccccc}
\hline$\mu$ & $A_{11}$ & $A_{12}$ & $A_{13}$ & $A_{14}$ & $A_{15}$ & $A_{16}$ & $A_{17}$ & $A_{18}$ & $A_{19}$ \\
\hline 0.01 & 0 & 0 & 0 & 0 & 0 & 0 & 0 & 0 & 0 \\
0.012149 & -0.004992 & -0.025728 & -0.052220 & -0.260352 & -0.520704 & -2.604288 & -5.208192 & -26.02368 & -52.003968 \\
0.015 & -0.011904 & -0.061056 & -0.122496 & -0.612864 & -1.226112 & -6.131328 & -12.262272 & -61.27296 & -122.445312 \\
0.0 & -0.024960 & -0.125184 & -0.250368 & -1.252224 & -2.504064 & -12.52032 & -25.038336 & -125.111424 & -250.020864 \\
0.03 & -0.051840 & -0.260736 & -0.521472 & -2.608512 & -5.217024 & -26.083968 & -52.164480 & -260.660736 & -520.917120 \\
0.05 & -0.112512 & -0.564480 & -1.129728 & -5.649408 & -11.298816 & -56.491776 & -112.975488 & -564.553728 & -1128.293376 \\
0.1 & -0.306816 & -1.535232 & -3.071232 & -15.355776 & -30.711168 & -153.548544 & -307.079040 & -1534.652160 & -3067.446528 \\
0.2 & -0.918528 & -4.593408 & -9.186816 & -45.933696 & -91.866624 & -459.317760 & -918.595200 & -4591.35936 & -9178.672128 \\
0.3 & -1.946496 & -9.732864 & -19.465728 & -97.329024 & -194.657664 & -973.262592 & -1946.46144 & -9729.753984 & -19453.120900 \\
0.4 & -3.620352 & -18.102912 & -36.206218 & -181.03104 & -362.060928 & -1810.268928 & -3620.449152 & -18098.68262 & -36188.455300 \\
\hline
\end{tabular}

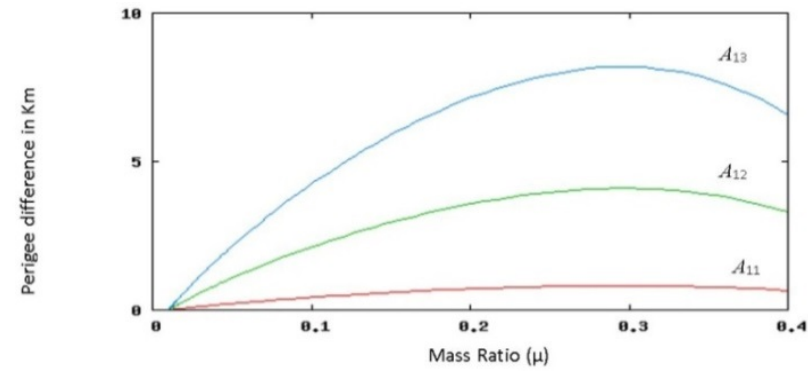

Figure 1. Perigee variations for various oblateness coefficients $A_{11}=0.000001, A_{12}=0.000005, A_{13}=0.00001$ when $p=0.23802754$ Vs mass ratio $(\mu)$.

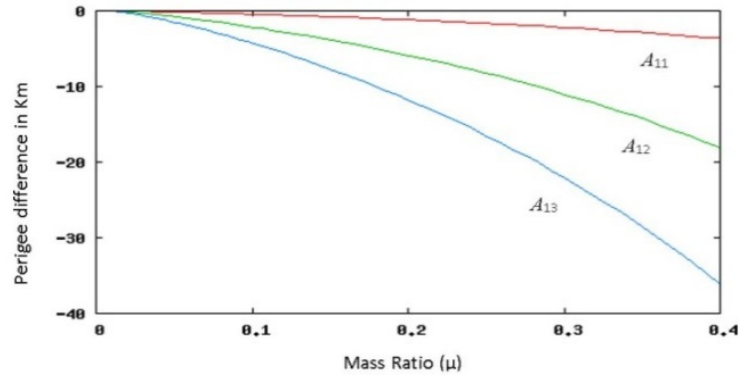

Figure 2. Perigee variations for various oblateness coefficients $A_{11}=0.000001, A_{12}=0.000005, A_{13}=0.00001$ when $p$ $=0.59999666 \mathrm{Vs}$ mass ratio $(\mu)$ 
Table 10. Difference in $x_{0}$ with and without oblateness for various periods $(\mu=0.012149$, each deviation multiplied by 3840000$)$.

\begin{tabular}{ccccccccc}
\hline$A_{1}$ & $T_{1}$ & $T_{2}$ & $T_{3}$ & $T_{1}$ & $T_{5}$ & $T_{6}$ & $T_{7}$ & $T_{8}$ \\
\hline 0 & 0 & 0 & 0 & 0 & 0 & 0 & 0 & 0 \\
$3.7 \mathrm{E}-07$ & 38.4 & 0 & 1.536 & -1.536 & -1.92 & -7.68 & 0 & 0.384 \\
$5 \mathrm{E}-07$ & 38.4 & 0 & 2.304 & -1.92 & -2.688 & -11.52 & 0 & 0.768 \\
0.000001 & 76.8 & 0 & 4.608 & -3.84 & -4.992 & -26.88 & -0.384 & 1.536 \\
0.000002 & 192.0 & 38.4 & 9.216 & -7.296 & -9.6 & -57.6 & -0.768 & 2.688 \\
0.000005 & 422.4 & 115.2 & 23.424 & -17.664 & -23.808 & -142.08 & -1.536 & 6.912 \\
0.00001 & 844.8 & 268.8 & 46.464 & -34.944 & -47.232 & -288 & -3.456 & 13.44 \\
0.00002 & 1689.6 & 576 & 93.312 & -69.888 & -94.08 & -579.84 & -6.912 & 26.88 \\
0.00005 & 4147.2 & 1459.2 & 233.472 & -174.336 & -235.008 & -1451.52 & -17.28 & 67.2 \\
0.0001 & 8332.8 & 2918.4 & 466.944 & -348.672 & -470.016 & -2903.04 & -34.944 & 134.016 \\
\hline
\end{tabular}

Table 11. Value of $x_{0}$ from $T=0.68419$ to 0.684208 .

\begin{tabular}{ccccccc}
\hline$A_{1}$ & $T_{19}$ & $T_{20}$ & $T_{201}$ & $T_{202}$ & $T_{203}$ & $T_{204}$ \\
\hline 0.000000 & -0.2119969670 & -0.2119988323 & -0.2119990211 & -0.2119992098 & -0.2119993986 & -0.2119995762 \\
0.00000037 & -0.211996967 & -0.2119988323 & -0.2119990211 & -0.2119992098 & -0.2119993986 & 0.2119995762 \\
0.00000050 & -0.211996967 & -0.2119988323 & -0.2119990211 & -0.2119992098 & -0.2119993986 & 0.2119995762 \\
0.00000100 & -0.211996967 & -0.2119988323 & -0.2119990211 & -0.2119992098 & -0.2119993986 & 0.2119995762 \\
0.00000500 & -0.211996967 & -0.2119988323 & -0.2119990211 & -0.2119992098 & -0.2119993986 & 0.2119995762 \\
0.00001000 & -0.211996967 & -0.2119988323 & -0.2119990211 & -0.2119992098 & -0.2119993986 & 0.2119995762 \\
0.00005000 & -0.211996967 & -0.2119988323 & -0.2119990211 & -0.2119992098 & -0.2119993986 & 0.2119995762 \\
0.00010000 & -0.211996967 & -0.2119988323 & -0.2119990211 & -0.2119992099 & -0.2119993986 & 0.2119995763 \\
\hline
\end{tabular}

Table 11(continued). Value of $x_{0}$ from $T=0.68419$ to 0.684208

\begin{tabular}{ccccccc}
\hline$A_{1}$ & $T_{205}$ & $T_{2057}$ & $T_{2059}$ & $T_{206}$ & $T_{207}$ & $T_{208}$ \\
\hline 0.00000000 & .2119998760 & -.2119998982 & -0.2119999315 & -0.2119999537 & -0.2120001425 & 0.2120003201 \\
0.00000037 & -0.2119998760 & -0.2119998982 & -0.2119999315 & -0.2119999537 & -0.2120001425 & -0.2120003201 \\
0.00000050 & -0.2119998760 & -0.2119998982 & -.2119999315 & -0.2119999537 & -0.2120001425 & 0.2120003201 \\
0.00000100 & -0.2119998760 & -0.2119998982 & -0.2119999315 & -0.2119999537 & -0.2120001425 & 0.2120003201 \\
0.00000500 & -0.2119998760 & -0.2119998982 & -0.2119999315 & -0.2119999537 & -0.2120001425 & 0.2120003201 \\
0.00001000 & -0.2119998760 & -0.2119998982 & -0.2119999315 & -0.2119999537 & -0.2120001425 & 0.2120003201 \\
0.00005000 & -0.2119998760 & -.2119998982 & -.2119999315 & -.2119999538 & -0.2120001423 & 0.2120003202 \\
0.00010000 & -0.2119998760 & -.2119998982 & -.2119999316 & -.2119999538 & -0.2120001425 & 0.2120003202 \\
\hline
\end{tabular}




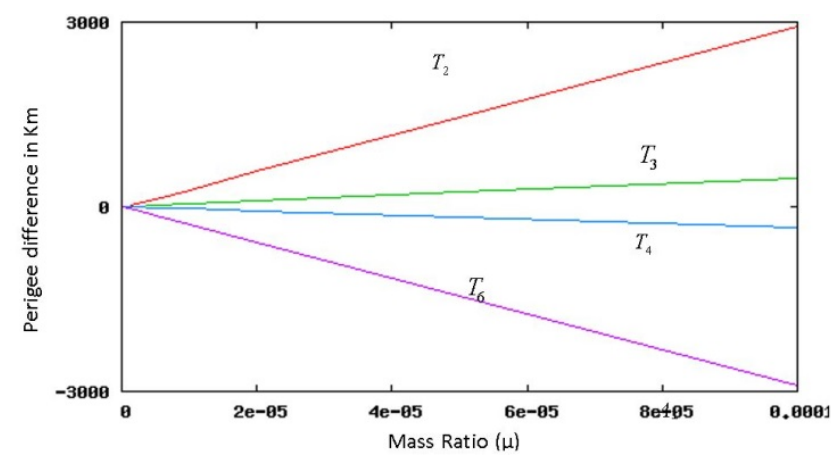

Figure 3. Perigee variations for periods $T_{2}=0.039999890, T_{3}=0.59999666, T_{4}=0.79999290, T_{6}=1.1999940$ with mass ratio $\mu=0.012149$.

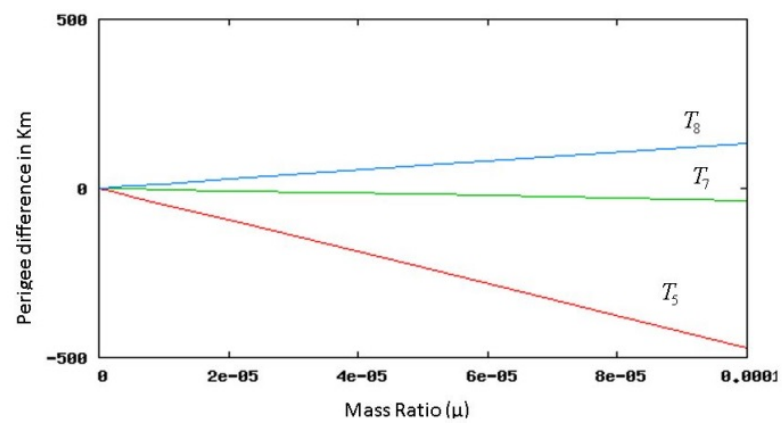

Figure 4. Perigee variations for periods $T_{5}=0.99999026, T_{7}=1.4000154, T_{8}=1.6000716$ with mass ratio $\mu=$ 0.01214 .

Table 12. Difference in $y_{0}$ with oblateness from that without oblateness for various periods.

\begin{tabular}{ccccccccc}
\hline$A_{1}$ & $T_{1}$ & $T_{2}$ & $T_{3}$ & $A_{1}$ & $T_{5}$ & $T_{6}$ & $T_{7}$ & $T_{8}$ \\
\hline 0 & 0 & 0 & 0 & 0 & 0 & 0 & 0 & 0 \\
$\mathrm{E}-07$ & 6.345 & 4.042 & 3.102 & 2.773 & 2.585 & 2.538 & 2.538 & 2.538 \\
$5 \mathrm{E}-07$ & 8.601 & 5.452 & 4.23 & 3.76 & 3.525 & 3.431 & 3.431 & 3.431 \\
0.000001 & 17.202 & 10.951 & 8.46 & 7.52 & 7.05 & 6.909 & 6.862 & 6.909 \\
0.000002 & 34.357 & 21.855 & 16.967 & 15.04 & 14.147 & 13.771 & 13.724 & 13.865 \\
0.000005 & 85.869 & 54.708 & 42.441 & 37.6 & 35.344 & 34.451 & 34.31 & 34.639 \\
0.00001 & 171.785 & 109.416 & 84.929 & 75.2 & 70.735 & 68.949 & 68.62 & 69.325 \\
0.00002 & 343.523 & 218.879 & 169.905 & 150.353 & 141.517 & 137.851 & 137.24 & 138.65 \\
0.00005 & 858.737 & 328.295 & 424.786 & 375.906 & 353.769 & 344.651 & 343.1 & 346.672 \\
0.0001 & 1717.427 & 1094.348 & 849.572 & 751.765 & 707.538 & 689.302 & 686.2 & 693.34 \\
\hline
\end{tabular}

\section{Conclusions}

In this paper, a series solution is developed for finding the initial conditions of periodic orbits by including oblateness effect of the more massive primary with the help of a perturbation technique. The results are verified with Huang's values. The effect of oblateness of the more massive primary on the initial conditions of the periodic orbits is studied by taking into consideration the variation of mass ratio $(\mu)$. As the mass ratio increases up to 0.012 , the perigee of the orbit moves towards the more massive primary. With the increase in oblateness coefficient, the perigee of the orbit shifts towards both the primaries depending upon the increase in period and mass ratio. It is further noticed that during this transition, for certain periods, the perigee of the orbit remains unaltered with the increase in the 
oblateness coefficient. The present study will be useful in understanding the general behavior of the motion of the inner planets and asteroids as a result of the perturbation by the major planets.

\section{References}

1. E. Stromgren, "Connaissance Actualle des Orbites dans le Problem des Trois Corps" Publications and Minor communications of Copenhagen Observatory, Publication 100, Copenhagen University, Astronomical Observatory, Denmark, 1935.

2. F. R. Moulton, "Periodic orbits", Carnegie Institute of Washington Publications, No.161, pp. 299-324, 1920.

3. G. H. Darwin, "Periodic orbits", Scientific Papers, Cambridge University Press, Cambridge, Mass, Vol 4, 1911.

4. R.A. Broucke, "Periodic orbits in the restricted three-body problem with Earth- Moon masses". Jet Propulsion Lab. Technical Report, 32-1168, Pasadena, CA, 1968.

5. V. G. Szebehely, "Theory of orbits", Academic press, New York, 1967.

6. S. W. McCuskey, "Introduction to Celestial Mechanics", New-York: Addison - Wesley, 1963.

7. A. H. Nayfeh, "Introduction to Perturbation Techniques", John Wiley and Sons, Inc. New York, 1981.

8. J. Guckenheimer and P. Holmes, "Nonlinear oscillations, dynamical systems, and bifurcations of vector fields", Springer-Verlag, New York, 1983.

9. J. W. Jordan, and P. Smith, "Nonlinear ordinary differential equations. An introduction to dynamical systems", 3rd ed. Oxford University Press Inc., New York, 1999.

10.J. Pelaez and M. Lara, "Periodic solutions in Electrodynamic Tethers on inclined orbits", Journal of Guidance, Control, and Dynamics, vol. 26. no.3, pp. 395-406, 2003.

11.S. S. Huang, "Preliminary study of orbits of interest for Moon Probes" Astron. J., vol. 67, pp 304-310, 1962.

12.S. S. Huang and C. Wade Jr., "Preliminary study of periodic orbits of interest for Moon Probes. II", Astron J., vol. 68, pp. 388-391, 1963.

13.S. S. Huang, "A Series solution for some periodic orbits in the restricted three-body problem according to the perturbation method", Technical Report, Goddard Space Flight Centre, NASA technical note, D-2488, 1964.

14.E. I. Abouelmagd, M. S. Alhothuali, Juan L. G. Guirao, and H. M. Malaikah, "Periodic and secular solutions in the restricted three-body problem under the effect of zonal harmonic parameters", Appl. Math. Inf. Sci., vol. 9, no. 4, pp. 1659-1669, 2015.

15.R. K. Sharma and P. V. Subba Rao, "Stationary solutions and their characteristic exponents in the restricted three-body problem when the more massive primary is an oblate spheroid" Celestial Mechanics, vol. 13, pp. 137149,1976 